\title{
Neighbor Selection Protocol for Heterogeneous Information Dissemination in Opportunistic Networks
}

\author{
*Halikul Lenando, ${ }^{+}$Abdallah Hassan Gharin, ${ }^{\ddagger}$ Mohamad Nazim Jambli, ${ }^{\S}$ Johari Abdullah, ${ }^{\ddagger}$ Adnan Shahid Khan \\ Faculty of Computer Science and Information Technology, \\ Universiti Malaysia Sarawak, Malaysia \\ Email: *cool@fit.unimas, ${ }^{*}$ dullmagic@gmail.com, ${ }^{{ }_{j}}$ jmnazim@fit.unimas.my, ${ }^{\S}$ ajohari@fit.unimas.my, ${ }^{\ddagger}$ skadnan@fit.unimas.my
}

\begin{abstract}
In Opportunistic Networks, information forwarding takes place when nodes are in communication range. Absence of knowledge on network topology prior information forwarding poses a compelling challenge in Opportunistic Networks. In these networks, disconnected mobile peers use mobility to opportunistically and dynamically connect to each other in order to disseminate heterogeneous information towards the intended destination. Flooding disseminates information to all nodes in range. Hence, it achieves high delivery performance. However, this form of dissemination congests the network and create considerable amount of network overheads. In this paper, a social-based neighbor selection protocol is proposed to enhance the heterogeneous information dissemination through peer-topeer interaction in Opportunistic Networks. Neighbor is selected based on social structure formulated by Frequency in Range, Frequency of Interaction metrics and Heterogeneity of information. A high valued metric peer from the list of candidate peers is selected as potential forwarder to disseminate information. The delivery performance impact of our protocol on information dissemination was investigated and has been analyzed in customized simulation tool. Experimental results showed that, the proposed neighbor selection protocol achieved delivery performance close to random selection in flooding and reduced $88.25 \%$ push overhead incurred during dissemination.
\end{abstract}

Keywords - context information, frequency in range, frequency of interaction, heterogeneous information, delivery performance, information dissemination, neighbor selection, opportunistic network, peer-to-peer interaction.

\section{INTRODUCTION}

In Opportunistic Networks, routes are evolved when messages are forwarded; this led to mixing together conceptual terms of routing and forwarding hence making those two terms to be used interchangeably [1]. Absence of network topology knowledge in Opportunistic Network makes routing the most compelling challenge [1]. In Opportunistic Networks, the disconnected "islands" of users are connected through node mobility, this makes end-to-end communications in such networks supported naturally. While other infrastructure network such as Mobile Ad Hoc Networks (MANET) treats the absence of network topology as an exception that needs some counter measures, Opportunistic Networks treat the same as natural state of the network by employing nodes' mobility to connect nodes opportunistically [2]. Because the communication path is opportunistically discovered on-the-fly due to absence of network infrastructure from source to destination node in advance, Epidemic Routing [3] is appropriate forwarding technique for such set-up as it does not only maximize message delivery rate and minimize message latency but also minimizes the total resources (e.g., memory and network bandwidth) used while delivering the message [3]. To make use of epidemic protocol's high delivery ratios and minimum message latency, social information about nodes' behavior is crucial in order to select the best forwarder of heterogeneous data in Opportunistic network. Mobile devices through their social behaviors depict specific movement patterns in the network which serve as context information for anticipating co-location and future contacts of nodes [5]. Incorporating social relationship between neighboring nodes with flooding protocol in order to be able to select appropriate node to forward information to take advantage of any future encounter is a very promising research direction for Opportunistic networks. Therefore, an efficient social relationship neighbor selection based on node's interaction history must be designed and investigated. In order to disseminate information between mobile nodes in Opportunistic networks [1], store-carry-and-forward paradigm [4] is used to forward data to the destination [6]. Node's identification process is based on the context information of the appropriate node [6]. In this paper we studied the impact of neighbor selection on the heterogeneous information dissemination performance through $\mathrm{P} 2 \mathrm{P}$ interactions in opportunistic networks.

The rest of the paper is organized as follows. Section II provides an overview of routing protocols in Opportunistic Network. Our proposed neighbor selection protocol is introduced in Section III. The simulation environment and corresponding performance evaluation of the neighbor selection technique is shown in Section IV. Finally, research work is concluded in section $\mathrm{V}$.

\section{RELATED WORK}

Opportunistic network is formed by wireless connected nodes. This formation is pair-wise, and takes place when two nodes are in communication range. Basically these nodes are devices equipped with short-range wireless communication capabilities. Node mobility and node activation/deactivation cause the connection to be temporary and thus as a result alter the network topology unpredictably. In Opportunistic 